\title{
In vitro evaluation of the antibacterial activity of modified multi-walled carbon nanotubes with phenolic extracts
}

https://doi.org/10.33263/BTEB214.017023

\author{
Mahdieh Entezari ${ }^{1,}{ }^{*}$, Zohreh Ghazi Tabatabaei $^{2}$, Amin Azarioun ${ }^{3}$, Sanaz Sarabian ${ }^{3}$, Ghazal \\ Taghizadeh Farahani ${ }^{3}$
}

1 Young researchers and elite club, Pharmaceutical sciences branch, Islamic azad University, Tehran, Iran

2 Department of chemistry, Ahar Branch, Islamic azad university, Ahar, Iran

3 Department of chemistry, Pharmaceutical Sciences Branch, Islamic azad university, Tehran, Iran *corresponding author e-mail address: mahdiehentezari63@yahoo.com

\section{ABSTRACT}

Conventional Antibiotics such as ciprofloxacin, ofloxacin and levofloxacin are an important group of antimicrobials which are widely used in the treatment of various infectious diseases. Long-term treatment with antibiotics can cause side effects in consumers. Bactericidal antibiotics may persuade the establishment of toxic reactive oxygen types (ROS) in bacteria. In the present study, we tried to eliminate the side effects of antibiotics by modification of multi wall carboxylated nanotubes with phenolic extractives that are extracted of pigmented vegetables. Very low concentrations of functionalized nanotubes were tested against both Gram-positive and Gram-negative bacteria. Antibacterial activity of the products was compared with MIC values of conventional antibiotics. In vitro studies represented that killed bacteria in the presence of low concentrations of modified nanotubes. so, the extremely abilities of functionalized carboxylated multiwall nanotubes have the potential to be described as innocuous antibiotic candidates.

Keywords: Antibacterial activity, MWNTs, Phenolic extractives, Modification.

\section{INTRODUCTION}

Antibiotics are a group of medicines that are synthesized to treat infections caused by bacteria and certain parasites. Antibiotics Antibiotics have led to an admirable reduction in diseases and fatality connected with bacterial infections. Freely prescribe antibiotics by doctors may seem like an advantage of antibiotics between drugs but the reported side effects of antibiotics, such as diarrhea, impaired kidney function, and inflammation of the tendons shows that prescription and the immethodical use of antibiotics should be controlled. Germicidal and antibacterial antibiotics have been shown to target mitochondrial components. Studies on mammalian cells treated with high concentrations of antibiotics have shown that these cells are destroyed and their growth is inhibited and have lost their metabolic activity [Armenise et al., 2012; Lawrence et al., 1996; Duewelhenke et al., 2007]. In other words, clinical levels of antibiotics can cause oxidative stress that can lead to damage to DNA, proteins and lipids in human cells, but this effect can be alleviated by antioxidants. For this reason, scientists in many cases, suggest diets rich in antioxidants as an alternative medicine, In fact, it seems that finding a material with anti-bacterial and antioxidant properties is very valuable. Many of the biological properties are closely associated with the antioxidant activity of anthocyanin pigment [Prior et al., 1998]. The antioxidative phytochemicals, especially phenolic compounds, found in vegetables and fruits have received increasing attention for their potential role in the prevention of human diseases [Abdel-Hameed et al., 2009; Cai et al., 2004; Dib et al., 2013]. The poly phenolic compounds are an important class of antioxidants, the polyphenols can be classified into ten classes. Flavonoids constitute the most common and widely distributed group of plant phenolics. Anthocyanins constitute the most 
conspicuous group of flavonoids. Natural sources of anthocyanins include a variety of colored fruits, vegetables, spices and nuts [Delgado-Vargas et al., 2000]. Outer skin of red onion is red, eggplant skin is violet and outer layers of red cabbage is red due to the presence of anthocyanins [Sadilova et al., 2006]. Nowadays, nanotubes are attractive compounds for biological targets. Nanotubes can be carriers of drugs or can be part of a drug [Cirillo et al., 2013].There are studies that were confirmed the antimicrobial activity of nanotubes and fullerenes [Kang et al., 2007; Patel et al., 2013], it is known that functionalization of nanotubes by association with organic compounds was proposed as an efficient strategy to increase their bio capability. But there are problems about toxicity of nanotubes and their negative effects on healthy cells and tissues. However, the studies have shown that the toxicity of nanotubes decreases if the functionalized multi wall carbon nanotubes are well dispersed in water [Vittorio et al., 2011]. For this purpose should be modified by compounds that make hydrogen bonds with water. Definitely phenolic anthocyanins are suitable for this purpose. Therefore in this study, we have tried to synthesized compounds that at very low concentrations are effective on bacteria and have not side effects of antibiotics used in clinical treatment. For this purpose, we extracted the extracts of skin of red onion, eggplant skin and outer layers of red cabbage because extracts of the veggies is rich in phenolic anthocyanins [Gachovska et al., 2010; Skerget et al., 2009; Saraf et al., 2009]. We did not separate the components of the extracts because natural phenolic agents from plants may have a major advantage since they might contain a spectrum of phenolic antimicrobials [Randhir et al., 2004]. Then we used the Fischer Esterification method for functionalization of MWNT-COOH with phenolic anthocyanins. The functionalization was approved by chemical spectra and solubility test that in this research is very important. The antibacterial activities of synthesized compounds were evaluated by effects of very low concentrations by using agar dilution method. The results were compared with the lowest concentration effects of current antibiotics.

\section{MATERIALS AND METHODS}

\section{Materials for extraction and modification}

All chemicals and reagents were purchased from Merck and Sigma-Aldrich and used without further purification. MWNT-COOH (95\% purity, 20-30 nm; Netrino Co. Ltd) were purchased and used as received.

\section{Experimental methods}

As anthocyanins are located inner the plant cell, in plant cells, organic solvents used for the extraction of homogeneous plant material. In the conventional extraction method organic solvents such as methanol, ethanol or acetone are used. Intracellular anthocyanins are released into the solvents but keep the anthocyanins in their native form. In this study, we have used ethanol for the extraction of anthocyanins of red onion skins, eggplant skin and the outer layers of red cabbage. In the past, usually for antioxidants extraction was used Soxhlet extraction method but now novel methods such as microwave assisted extraction, ultrasound assisted extraction, pulsed electric field assisted extraction and high hydrostatic pressure are used instead of the old method. But generally, temperatures higher than $70^{\circ} \mathrm{C}$ cause rapid degradation and discoloration of anthocyanins [Kirca et al., 2003]. Light is a significant factor accelerating anthocyanin degradation similar to temperature [Chiste et al., 2010]. therefore, we apperceive that ultrasonic assisted extraction is discovered as an alternative method which can be used to replace traditional methods and undesirable new methods. The ultrasonic assisted extraction is a greener method that allows for high reproducibility in a shorter time with simple manipulation and with a significant reduction in the consumption of organic solvent and temperature and is associated with less input energy[Ma et al, 2011; Tadeo et al., 2010; Sisti et al., 2008; Shahat et al., 2011]. Due to the advantages of this method we used for 
anthocyanins extraction of ultrasonic method. Three grams of each plant sample was washed with water, then completely was dried and $30 \mathrm{ml}$ of ethanol was added. The mixtures were placed in an ultrasonic bath and were sonicated at room temperature for one hour. The contents of each container were filtered and had been for a week at room temperature in a dry place away from light. Extracts that has anthocyanins were extracted from each sample.

\section{Preparation of modified nanotubes}

0.005 grams of MWNT-COOH (with >95\% purity were purchased of notrino company) for further shortening were sonicated with $10 \mathrm{cc}$ of normal hexane and were sonicated with extracts that have anthocyanins for one hour at room temperature. Sonication also helps in more solubilization of nanotubes in aques media then $0.5 \mathrm{cc}$ of concentrated sulfuric acid was added to each mixture as catalyst. Moreover, this step also leads to some sidewall functionalization as strong strength of acids was used .The mixtures were stirred at room temperature for $96 \mathrm{~h}$. Each sample was then filtered and washed with water and ethanol. The products were then dried at room temperature for 20 hours (Figure. 1).

MWNT-COOH $\stackrel{\text { Sonicaton }}{\longrightarrow}$ Shorter nanotubes $\frac{\text { Phenolic extracts }}{\mathrm{H}_{2} \mathrm{SO}_{4}}$ Natural Nano Products

Figure 1. Formation of the Natural Nano Compounds.

\section{In vitro studies}

Tested microorganisms:

For the purpose of antibacterial evaluation of the functionalized nannotubes, tow bacterial species, Pseudomonas (Gram-negative), Staphylococcus aureus (Gram-positive) were used.

Antibacterial activity:

The antibacterial activity of nano compounds was determined against Gram-positive and Gramnegative bacteria. The inhibitory concentrations of compounds were determined by the conventional agar dilution method. The bacterial species were cultured on nutrient agar media, each bacterial species was incubated for $48 \mathrm{~h}$ at $37^{\circ} \mathrm{C}$. Then half McFarland standard was prepared from bacteria. For preparation of nano solution, nano specimens were washed with water and alcohol. The remaining solid samples were dissolved in $\mathrm{H} 2 \mathrm{O}$ and then were diluted with distilled water to obtain the required concentrations of 0.0036 and $0.006 \mu \mathrm{g} / \mathrm{mL}$. The bacteria were dispensed into medium in sterile Petri dish. Then, the nano samples were with well method in Petri dishes. Petri dishes were inoculated at $37 \mathrm{OC}$ for $24 \mathrm{~h}$. The inhibitory concentration was defined of the test compounds, which resulted in no visible growth on the plate.

\section{RESULTS AND DISCUSSION}

One of the best methods to confirm the functionalization of nanotubes is the FT-IR spectra. In the spectrum of MWNT-COOH the characteristic absorption peak of $\mathrm{C}=\mathrm{O}$ appear at $1644 \mathrm{~cm}-1$ but in the spectra of the products the stretching peak of carbonyl was observed at 1650, 1644 and 1645 $\mathrm{cm}-1$ for $B, C$ and $D$ respectively. The shift of the carbonyl peak to higher wavelengths is an approval for the esterification of the nanotube surface in $B$ and $D$. The strong peaks are observed in around $1200-1100 \mathrm{~cm}-1$ that are relevant to $C$ $O$ bond in esters, thus ester formation is confirmed. On the other peaks at about 1458, $1461,1515 \mathrm{~cm}-1$ are seen in the product spectra which can be related to the stretching vibrations of the ring flavonoids [Merlin et al., 1994]. The appearance of the increased intensity of the peak around $2900 \mathrm{~cm}-1$ after surface treatment of MWNT-COOH confirms the incorporation of flavonoid groups. (Table1).

Table 1. Diagnostic IR bands of the MWNT-COOH and Functionalized MWNTs with anthocyanins.

\begin{tabular}{ccccccc}
\hline $\begin{array}{c}\text { Compound } \\
\text { name }\end{array}$ & $\begin{array}{c}v(\text { The } \\
\text { Acidic } \\
\mathrm{OH})\end{array}$ & $\begin{array}{c}v(\text { The } \\
\text { Phenolic } \\
\mathrm{OH})\end{array}$ & $\begin{array}{c}v(\mathrm{C}- \\
\mathrm{C}\end{array}$ & $\begin{array}{c}v \\
(\mathrm{C}=\mathrm{O})\end{array}$ & $\begin{array}{c}v(\mathrm{C}=\mathrm{C}) \\
\text { rings of } \\
\text { flavonoids }\end{array}$ & $\begin{array}{c}v(\mathrm{C}- \\
\mathrm{O})\end{array}$ \\
\hline A & 3741 & 3500 & 2918 & 1644 & - & - \\
B & - & 3400 & 2925 & 1650 & 1513,1461 & 1168 \\
C & - & 3400 & 2923 & 1644 & 1514.98 & 1200 \\
D & - & 3400 & 2925 & 1645 & 1458 & 1100 \\
\hline
\end{tabular}


Biomaterials and Tissue Engineering Bulletin

UV spectroscopy provides useful information about changes in the surface of nanotubes thus facilitate the identification of functionalized nanotubes. $0.001 \mathrm{~g}$ of each sample was dissolved in $50 \mathrm{ml}$ of water, then was sonicated for one hour and remained motionless until UV spectra were measured in the range of $200-800 \mathrm{~nm}$. The characteristic absorption peak of MWNT-COOH is showed at around $246 \mathrm{~nm}$ that is relating to $\pi \rightarrow \pi^{*}$ electron transition in $\mathrm{C}=\mathrm{O}$ which is conjugated with double bonds in the nanotube but this $\lambda$ max in modified nanotubes was changed because anthocyanins have been replaced instead hydroxyl group. Moreover if nanotubes are functionalized with anthocyanins, modified nanotubes have $\pi \rightarrow \pi^{*}$ and $n \rightarrow \pi^{*}$ electron transitions and in the UV spectra of the products are observed peaks in two separate bands that are related to transactions in anthocyanins structure. This absorptions are observed in the range between 240-289 $\mathrm{nm}$ which is known as the Band II and in the range between $300-400 \mathrm{~nm}$ which is called the Band I, it is clear that this value is somewhat and is related to the type of anthocyanins. The study of UV spectra of MWNT-COOH and nano products was appropriate approval for functionalization of MWNT-COOH with anthocyanins (Table2) [Sisa et al., 2010].

Table 2. UV-visible absorption peaks of MWNTs (A-D) in $\mathrm{H}_{2} \mathrm{O}$.

\begin{tabular}{cccc}
$\begin{array}{c}\text { Compound } \\
\text { name }\end{array}$ & $\begin{array}{c}\mathrm{n} \rightarrow \pi^{*} \\
(\mathrm{C}=\mathrm{O})\end{array}$ & $\begin{array}{c}\pi \rightarrow \pi^{*} \\
(\mathrm{C}=\mathrm{C}) \\
\text { bandII }\end{array}$ & $\begin{array}{l}\pi \rightarrow \pi^{*} \\
(\mathrm{C}=\mathrm{C}) \\
\text { bandI }\end{array}$ \\
\hline A & 246 & - & - \\
B & 244 & 302 & 380 \\
C & 245 & 271 & 286 \\
D & 248 & 259 & 384 \\
\hline
\end{tabular}

Functionalization was also confirmed by solubility test which is the most sensitive test available for this study. Since anthocyanins have functional groups that can make hydrogen bonds with water if nanotubes are functionalized with these natural products, these compounds are well dispersed in water, while the nanotubes with non-phenolic functional groups are very low dispersed in water. Prepared samples for antibacterial activity were used to detection the ability of disperse in water. For this purpose $1 \mathrm{mg}$ of each sample nanotube was mixed with $100 \mathrm{ml}$ of water, and was sonicated for one hour then dispersion of nanotubes was studied in consecutive days. It is observed that the dispersibility of MWNTs in water is remarkably changed after the modification of MWNT-COOH with anthocyanins [Sheikholeslam et al., 2012]. Studies in Figure 2 showed that even after 1 week, the dispersibility of the samples is still stable. Thus the solubility tests confirmed the functionalization of nanotubes with phenolic extracts. It is noteworthy that $D$ is exhibited stronger and better solubility of other products.

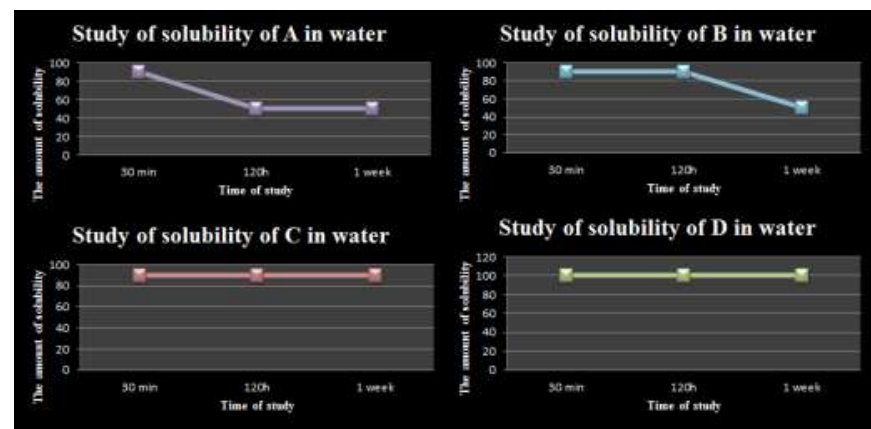

Figure 2. Solubility test of MWNT-COOH (A) and functionalized MWNTs (B-D). In this diagram, 100 is indicative of complete solubility and constant dispersion and the other values are expressed in comparison.

After the excellent solubility of functionalized nanotubes have been studied minimum inhibitory concentrations (MIC) of them on pathogenic micro-organisms.

Minimum inhibitory concentrations (MIC) of antibiotics were determined against Gram-positive (S. aureus) and Gram-negative (Pseudomonas) bacteria are showed in Figure 3 [Emami et al.,2013]. The concentration values of test compounds against bacteria were in the range of 0.0036 and $0.006 \mu \mathrm{g} / \mathrm{mL}$. Interesting results of bacterial growth were showed in the presence of modified nanotubes. Among the studied nano samples, compound $D$ was the most potent compound against bacterial growth. Compound $\mathrm{D}$ with MIC values nigh $0.006 \mu \mathrm{g} / \mathrm{mL}$ exhibited potent inhibitory activity against pseudomonas. Its activity was 65 -fold more than that of standard drug ciprofloxacin. Moreover, compound D showed a lot more power than norfloxacin that is usually prescribed. 
Biomaterials and Tissue Engineering Bulletin

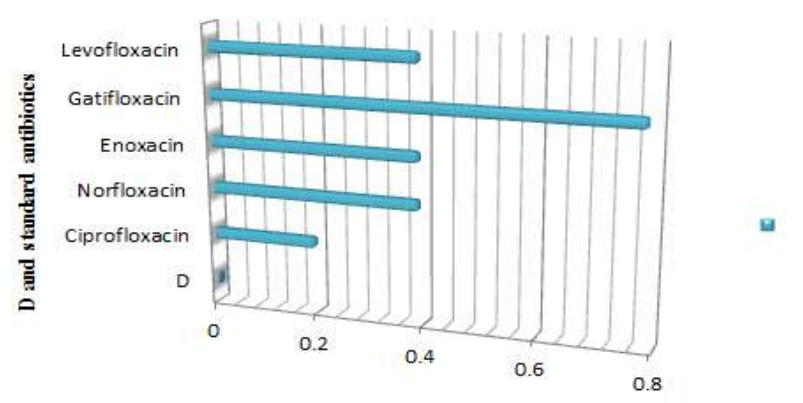

Comparision of MICs against Staphylococcus aureus

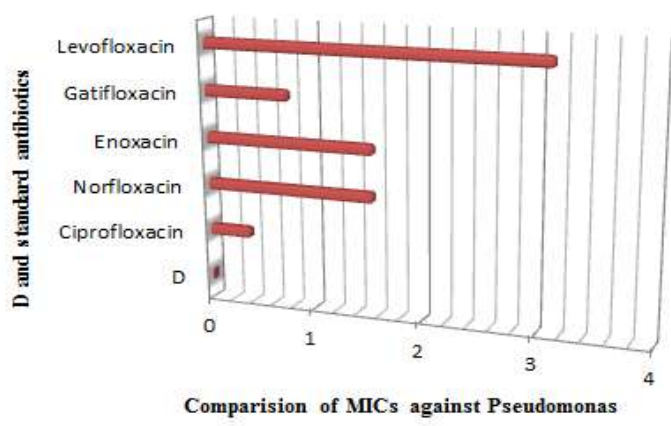

Figure 3. Evaluation of low concentration of compound $D$ in comparison with minimum inhibitory concentrations of standard antibiotics against pathogenic bacteria.
The comparison of the MIC values of norfloxacin, enoxacin and levofloxacin against pseudomonas were much less effective than the compound $D$. In the case of $S$. aureus, compound D with MIC value of $0.0036 \mu \mathrm{gg} / \mathrm{mL}$ was the most potent compounds, being 54 times more potent than ciprofloxacin. In addition, the activity of compound $D$ against $S$. aureus was very superior to that of norfloxacin, Enoxacin and Levofloxacin. The antibiotics are not effective in the very low concentrations that the modified nanotubes were studied, so to be effective in the treatment process must be used at higher doses and this can cause side effects. Many of these side effects will cause the patient is unable to continue taking the medication and this can cause serious complications in all organs of the body. But nano tubes by this research are showed one other of the their amazing properties. In fact, this study showed that compounds are made of nanotubes with natural compounds can make a big change in the treatment of infectious diseases.

\section{CONCLUSIONS}

The results of the studies showed replacement of phenolic flavonoids at $\mathrm{OH}$-position of $\mathrm{COOH}$ with phenolic $\mathrm{OH}$ of onion oxidants decreased the antibacterial activity against both Gram-positive and Gram-negative bacteria. By comparing the antibacterial activity of phenolic compounds in red onion skins (D) and antioxidants in eggplant skin and outer layers of red cabbage (B and $C$ ), it is revealed that the antioxidants in red onion skin are more favorable group. The introduction of the ester group at the external position of nanotubes by flavonoids of red onion skin could improve the antibacterial activity in very low concentration. Similarly, the comparison of compounds $B$ and $C$ demonstrated that substitution of phenolic extractives of eggplant skin and outer layers of red cabbage with ester band at external position of MWNT-COOH had no positive effect on activity, when in low concentrations were studied. The use of natural phenols in modification of nanotubes could be a suitable method to synthesize antibiotics with minimal side effects. This study could be the beginning of a major transformation in the synthesis of antibiotics that would reduce the suffering of patients with use of conventional antibiotics.

\section{REFERENCES}

Armenise, D., Muraglia, M., Florio, M. A., Laurentis, N. D., Rosato, A., Carrieri, A., Corbo, F., Franchini, C. (2012). 4H-1,4-Benzothiazine, Dihydro-1,4-benzothiazinones and 2-Amino-5-fluorobenzenethiol Derivatives:
Design, Synthesis and in vitro Antimicrobial Screening. Arch. Pharm: 345(5), 407-416.

Lawrence, J. W., Claire, D. C., Weissig, V. (1996). Delayed cytotoxicity and cleavage of mitochondrial DNA in 
ciprofloxacin-treated mammalian cells. Mol. Pharmacol. Mol. Pharmacol: 50, 1178-1188.

Duewelhenke, N., Krut, O., Eysel, P. (2007). Influence on mitochondria and cytotoxicity of different antibiotics administered in high concentrations on primary human osteoblasts and cell lines. Agents Chemother: 51, 54-63.

Prior, R.L., Cao, G., Martin, A., Sofic, E., McEwen, J., O'Brien, C., Lischner, N., Ehlenfeldt, M., Kalt, W., Krewer, G., Mainland, C. M. (1998). Antioxidant Capacity As Influenced by Total Phenolic and Anthocyanin Content, Maturity, and Variety of Vaccinium Species. J. Agric. Food Chem: 46, 2686 -2693.

Abdel-Hameed, El.S.S.( 2009). Total phenolic contents and free radical scavenging activity of certain Egyptian Ficus species leaf samples. Food Chem: 114 (4), 1271-1277.

Cai, Y., Luo, Q., Sun, M., Corke, H.( 2004). Antioxidant activity and phenolic compounds of 112 traditional Chinese medicinal plants associated with anticancer. Life Sci: 74, 2157-2184.

Dib, M.E.A., Allali, H., Bendiabdellah, A., Meliani, N., Tabti, B.( 2013). Antimicrobial activity and phytochemical screening of Arbutus unedo L. J. Saudi Chem. Soc: 17(4), 381-385.

Delgado-Vargas, F., Jiménez, AR., Paredes-Lopez, O. (2000). Natural pigments: carotenoids, anthocyanins, and betalains--characteristics, biosynthesis, processing, and stability. Crit. Rev. Food Sci. Nutr: 40(3), 173-289.

Sadilova, E., Stintzing, F. C., Carle, R. (2006). Anthocyanins, Colour and Antioxidant Properties of Eggplant (Solanum melongena L.) and Violet Pepper (Capsicum annuum L.) Peel Extracts. Z. Naturforsch: 61(C), 527535.

Cirillo, G., Vittorio, O., Hampelb, S., Spizzirri, U. G., Picci ,N., lemmaa, F. (2013). Incorporation of carbon nanotubes into a gelatin-catechin conjugate: Innovative approach for the preparation of anticancer materials. Int. J. Pharm: 446, 176- 182.

Kang, S., Pinault, M., Pfefferle, L.D., Elimelech, M. (2007). Single-Walled Carbon Nanotubes Exhibit Strong Antimicrobial Activity. Langmuir: 23, 8670-8673.

Patel, M. B., Harikrishnan, U., Valand, N. N., Modi, N. R., Menon, S. K.( 2013). Novel Cationic Quinazolin-4(3H)one Conjugated Fullerene Nanoparticles as Antimycobacterial and Antimicrobial Agents. Arch. Pharm: 346(3), 210-220.

Vittorio, O., Duce, S., Pietrabissa, A., Cuschieri, A. (2011). Multiwall carbon nanotubes as MRI contrast agents for tracking stem cells. Nanotechnol: 22, 095706.

Gachovska, T., Cassada, D., Subbiah, J., Hanna, M., Thippareddi, H., Snow, D. (2010). Enhanced Anthocyanin Extraction from Red Cabbage Using Pulsed Electric Field Processing. Journal of Food Science: 75 (6), E323-E329.

Skerget, M., Majheniè, L., Bezjak, M., Knez, Z. (2009). Antioxidant, Radical Scavenging and Antimicrobial
Activities of Red Onion (Allium cepa L) Skin and Edible Part Extracts. Chem. Biochem. Eng. Q: 23(4), 503510.

Saraf, P., Saraf, R. (2009). Integrated Approach in the Management of Chronic Heart Failure. Journal of The Fiji College of General Practitioners: 17(1), 6-7.

Randhir, R., Lin, Y.T., Shetty, K. (2004). Stimulation of phenolics, antioxidant and antimicrobial activities in dark germinated mung bean sprouts in response to peptide and phytochemical elicitors. Process Biochem: 39, 637-646.

Kirca, A., Cemeroglu, B. (2003). Degradation kinetics of anthocyanins in blood orange juice and concentrate. Food Chem: 81(4), 583-587.

Chiste, R. C., Lopes, A. S., Faria, L. J. G. D. (2010). Original article: Thermal and light degradation kinetics of anthocyanin extracts from mangosteen peel (Garcinia mangostana L.). Int. J. Food Sci. Technol: 45, 19021908.

Ma, C.H., Liu, T.T., Yang, L., Zu, Y.G., Wang, Sh.Y., Zhang, R.R. (2011). Study on ionic liquid-based ultrasonic-assisted extraction of biphenyl cyclooctene lignans from the fruit of Schisandra chinensis Baill. Anal. Chim. Acta: $689,110-116$.

Tadeo, C., Sanchez, B., Albero, A.I., Garcia,V. (2010). Application of ultrasound-assisted extraction to the determination of contaminants in food and soil samples. J. Chromatogr. A: 1217, 2415-2440.

Sisti, M., Santi, M. De., Fraternale, D., Ninfali, P., Scoccianti, V., Brandi, G. (2008). Antifungal activity of Rubus ulmifolius Schott standardized in vitro culture. LWTFood Science and Technology: 41, 946-950.

Shahat, A. A., Ibrahim, A. Y., Hendawy, S. F., Omer, E. A., Hammouda, F. M., Abdel-Rahman, F. H., Saleh, M. A. (2011). Chemical Composition, Antimicrobial and Antioxidant Activities of Essential Oils from Organically Cultivated Fennel Cultivars. Molecules: 16, 1366-1377.

Merlin, J.C., Cornard, J.P., Stastoua, A., Saidi-Idrissi, M. (1994). Vibrational analysis of hydroxyflavylium derivatives by IR, Fourier transform Raman and resonance Raman spectroscopies. Spectrochim. Acta: 50A, 703-712.

Sisa, M., Bonnet, S. L., Ferreira, D., Westhuizen, J.H.V.d. (2010). Photochemistry of Flavonoids. Molecules: 15, 5196-5245.

Sheikholeslam, M., Pritzker, M., Chen, P. (2012). Dispersion of multiwalled carbon nanotubes in water using ioniccomplementary peptides. Langmuir: 28 (34), 1255012556.

Emami, S., Shahrokhirad, N., Foroumadi, A., Faramarzi , M. A., Samadi, N., Soltani Ghofrani, N. (2013). Piperazinylquinolones with methylene-bridged nitrofuran scaffold as new antibacterial agents. Med Chem 22, $\quad$ 2940-5947. 


\section{ACKNOWLEDGEMENTS}

We are very grateful for the support of Young Researchers and Elite Club of Islamic Azad University of pharmaceutical sciences branch. We are also very grateful for the cooperation of Dr Fatemeh Ashouri, Dr Sepideh Arbabi, Dr Mahnaz Ghomi and Dr Setareh Haghighat.

\section{Conflicts of Interest}

The authors declare no conflict of interest.

(C) 2015 by the authors; licensee AMG Transcend, Bucharest, Romania. This article is an open access article distributed under the terms and conditions of the Creative Commons Attribution license (http://creativecommons.org/licenses/by/4.0/). 